\title{
Cardiorespiratory and Biomechanical Responses to Simulated Horseback Riding in Healthy Children
}

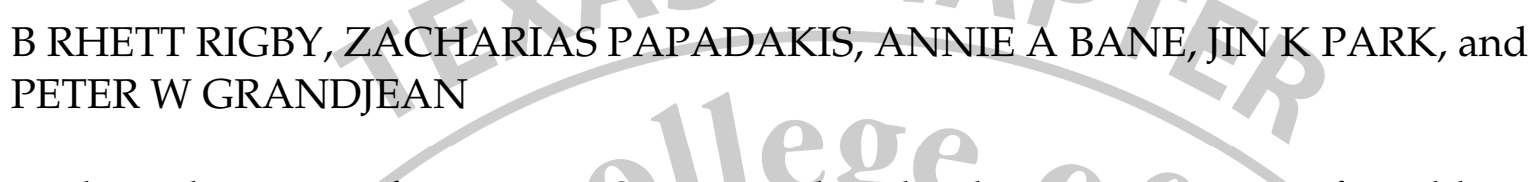

Baylor Laboratories for Exercise Science and Technology; Department of Health, Human Performance, and Recreation; Baylor University; Waco, TX

\section{Category: Doctoral}

The movement of specific body segments during horseback riding is similar to common physical activities such as walking. Yet, little is known regarding the cardiovascular responses to horseback riding (HBR) which may be due to how difficult it is to quantify the physical work of HBR. The purpose of this study was to characterize cardiorespiratory and pelvic kinematic responses to simulated horseback riding (SHR) in apparently healthy children. These responses were compared to walking on a treadmill at different intensities. Fifteen healthy children ( 8 females, 7 males; $9 \pm 3$ years of age; height $56 \pm 6$ in; weight $92.0 \pm 41.0$ $\mathrm{lb}$; body fat $27 \pm 8 \%$ ) walked at steady state on a treadmill (1-3 mph in $0.5 \mathrm{mph}$ increments, $0 \%$ grade) during one session and completed SHR [low intensity $(0.27 \mathrm{~Hz})$ and high intensity $(0.65 \mathrm{~Hz})$ ] during three sessions on differnet occasions. Physiologic variables, such as $\mathrm{HR}, \mathrm{VO}_{2}, \mathrm{~V}_{\mathrm{E}}, \mathrm{SBP}, \mathrm{DBP}$, were measured at rest and steady-state exercise at each intensity. Mean arterial pressure (MAP) and rate pressure product (RPP) were calculated. Pelvic kinematics were measured in a similar manner during SHR. Physiological variables across all three SHR sessions were similar at each intensity $(p>0.05)$. $\mathrm{VO}_{2}, \mathrm{~V}_{\mathrm{E}}, \mathrm{MAP}$ and RPP were greater with high-intensity riding compared to low-intensity riding and rest. Pelvic kinematics followed the same pattern and were reproducible across SHR sessions. Treadmill walking at all speeds elicited greater magnitudes in the physiological variables compared to SHR. The ICC for RPP, an important variable used to index cardiorespiratory fitness, was 0.057 indicating good reproducibility across SHR sessions. The results of the present study suggest that cardiorespiratory responses and pelvic kinematics are reproducible with SHR in young children and these responses were lower than those elicited by treadmill walking at $1 \mathrm{mph}$.

Table 1: Means of cardiorespiratory variables at rest and during simulated horseback riding

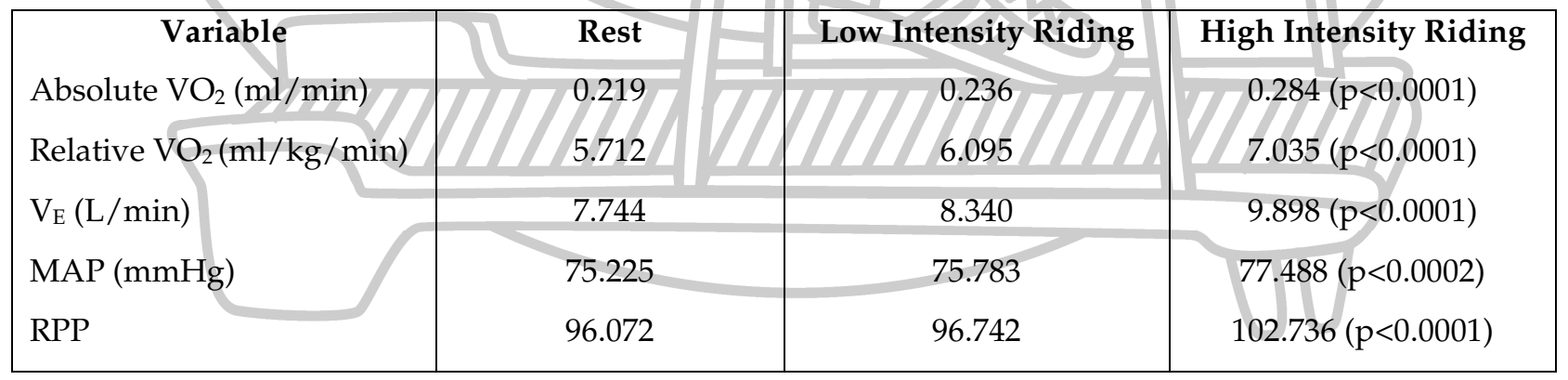




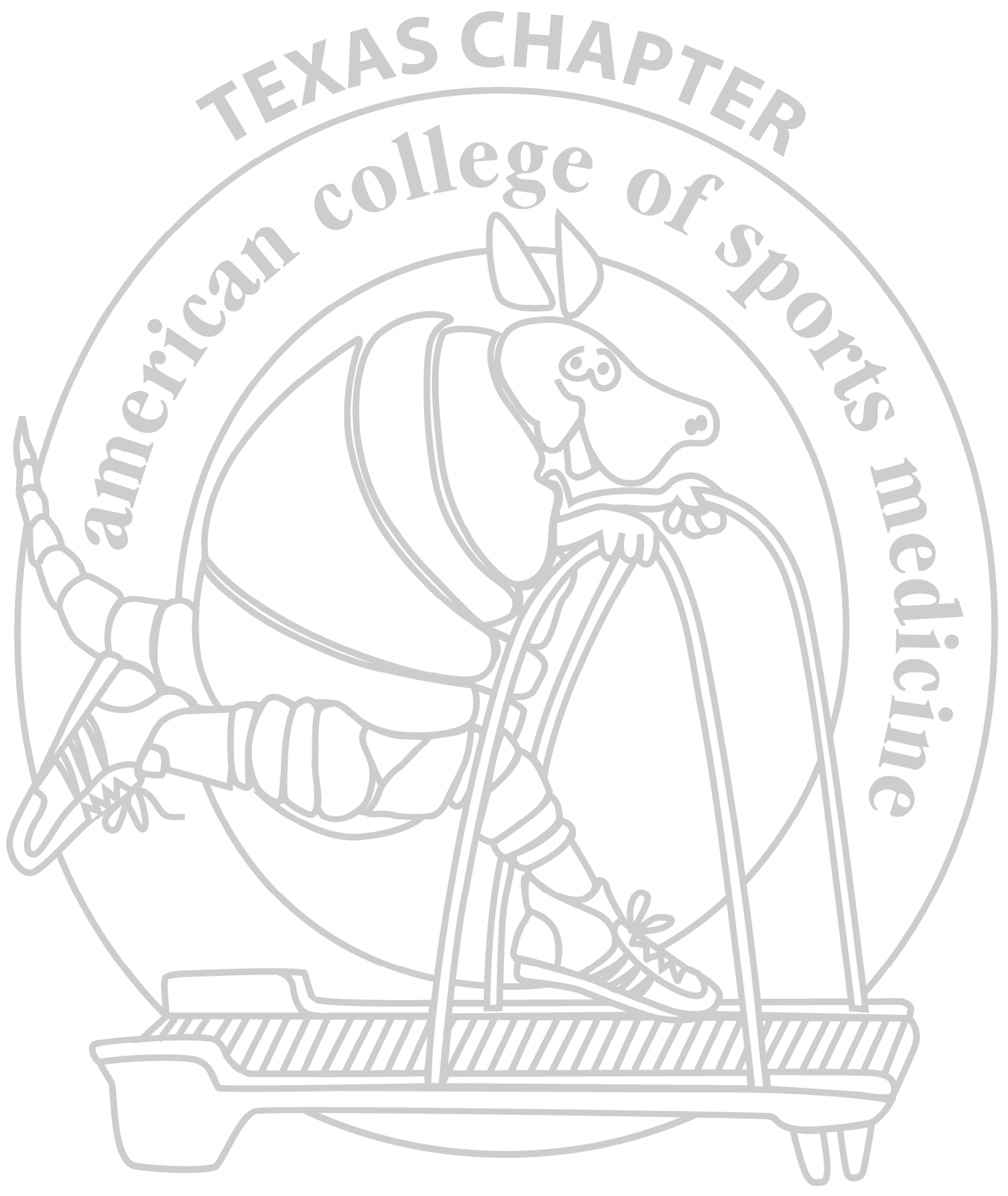

International Journal of Exercise Science

www.tacsm.org 